\title{
Programa Médicos pelo Brasil: inovação ou continuidade?
}

\section{Doctors throughout Brazil Program: innovation or continuity?}

\section{Programa Médicos en todo Brasil: ¿innovación o continuidad?}

\author{
Alexandre José de Melo Neto ${ }^{1} \mathbb{D}$, Danyella da Silva Barreto ${ }^{1}[$ \\ ${ }^{1}$ Universidade Federal da Paraíba (UFPB). João Pessoa, PB, Brasil.
}

\section{Resumo}

Apesar do lançamento do Programa Médicos pelo Brasil (PMB) alardear a ideia de inovação, este artigo evidencia o processo de continuidade do programa atual em relação ao Programa Mais Médicos (PMM). O PMB se estrutura nos acertos do PMM para montar o seu arcabouço de funcionamento e tenta superar problemas existentes no programa anterior. A principal proposta do PMB é a carreira médica para atuação na Atenção Primária à Saúde (APS), sem, no entanto, apresentar outros elementos importantes para a fixação profissional. Desta forma, apresenta-se como uma política mais frágil que seu antecessor, com foco apenas no provimento de médicos, correndo o risco de não atingir os objetivos a que se propôs enquanto política pública. Além disso, através da proposta de criação da Agência para o Desenvolvimento da Atenção Primária à Saúde (Adaps), o programa abre margem para a privatização dos serviços de APS e do Sistema Único de Saúde como um todo.

Palavras-chave: Política de Saúde; Atenção Primária à Saúde; Consórcios de Saúde; Reorganização de Recursos Humanos

\begin{abstract}
Despite the launch of the Doctors throughout Brazil Program (PMB) bring the idea of innovation, this article highlights the continuity of the current program in relation to the More Doctors Program (PMM). The PMB structures itself in the PMM's settings to assemble its operating framework and attempts to overcome existing problems in the previous program. The main proposal of the PMB is the medical career to work in Primary Health Care (PHC), without, however, presenting other important elements for professional fixation. Thus, it presents itself as a more fragile policy than its predecessor, focusing only on the provision of doctors, at the risk of not achieving the objectives it has set itself as a public policy. In addition, through the proposal to create the Agency for the Development of Primary Health Care (Adaps), the program leaves room for the privatization of PHC services and the Unified Health System as a whole.
\end{abstract}

Keywords: Health Policy; Primary Health Care; Health Consortia; Personnel Turnover

Como citar: Melo Neto AJ, Barreto DS. Programa Médicos pelo Brasil: inovação ou continuidade?. Rev Bras Med Fam Comunidade. 2019;14(41):2162. https://doi.org/10.5712/rbmfc14(41)2162
Autor correspondente: Alexandre José de Melo Neto. E-mail: halemao_jp@hotmail.com Fonte de financiamento: declaram não haver.

Parecer CEP: não se aplica.

Procedência e revisão por pares: revisado por pares. Recebido em: 07/08/2019. Aprovado em: 15/08/2019. 


\section{Resumen}

Aunque el lanzamiento del Programa Médicos en todo Brasil (PMB) promocionó la idea de innovación, este artículo destaca la continuidad del programa actual en relación con el Programa Más Médicos (PMM). El PMB se basa en los aciertos del PMM para construir su marco operativo e intenta superar los problemas existentes en el programa anterior. La propuesta principal del PMB es la carrera médica para trabajar en la Atención Primaria a la Salud (APS), sin presentar, sin embargo, otros elementos importantes para la fijación profesional. Por lo tanto, se presenta como una política más frágil que su predecesor, centrándose solo en la provisión de médicos, a riesgo de no lograr los objetivos que se ha fijado como política pública. Además, a través de la propuesta para la creación de la Agencia para el Desarrollo de la Atención Primaria a la Salud (Adaps), el programa deja margen para la privatización de los servicios de APS y el Sistema Único de Salud en su conjunto.

Palabras clave: Política de Salud; Atención Primaria de Salud; Consorcios de Salud; Reorganización del Personal

\section{O contexto do novo programa: Inovação ou Continuidade?}

Apesar do lançamento do Programa Médicos pelo Brasil (PMB) alardear a ideia de ruptura e inovação, o título deste artigo é intencional e tenta evidenciar o processo de continuidade do programa atual em relação ao Programa Mais Médicos (PMM) e seu eixo de provimento chamado de Projeto Mais Médicos para o Brasil (PMMB). ${ }^{1}$

É bem verdade que ainda é cedo para análises mais profundas sobre o 'novo' programa, já que os dados a serem utilizados para avaliação se baseiam principalmente na intencionalidade captada em declarações públicas e em uma medida provisória $(\mathrm{MP})^{2}$ que trata apenas parcialmente das medidas até então divulgadas. Ainda há muito para acontecer, inclusive com as disputas dentro do próprio Congresso que podem reconfigurar a MP como aconteceu na criação da lei do Mais Médicos. ${ }^{3,4}$

Feita esta ressalva, é importante começar destacando a centralidade da Atenção Primária à Saúde (APS) na pauta política do Ministério da Saúde (MS) atual, fazendo com que suas primeiras ações tenham este foco com a criação de uma Secretaria de Atenção Primária à Saúde ${ }^{5}$ e agora com um programa que tenta ganhar a mesma visibilidade e aceitação pública que o PMM. Além disso, o programa surpreende ao conseguir capitalizar recursos em um cenário de recessão e restrição orçamentária com desmonte de diversos políticas públicas devido à retomada da ortodoxia neoliberal ${ }^{6,7}$ e as políticas de austeridade ${ }^{8,9}$ merecendo, por isso, o seu merecido reconhecimento.

Poder-se-ia, a partir disso, de forma simplória, creditar este destaque somente ao trabalho do corpo técnico atual do Ministério da Saúde, mas é preciso destacar que há anos se vem construindo um contexto de fortalecimento da APS no Brasil, que deixa de ser tratada somente como programa e passa a se constituir como estratégia de reorientação do modelo assistencial já na década passada, ${ }^{10} \mathrm{com}$ diversas políticas orientadas para este fim na formação em saúde, ${ }^{11}$ na qualificação estrutural ${ }^{12,13}$ e com diversos resultados verificados na qualidade da assistência. ${ }^{14-21}$

Entretanto, mesmo com a diversidade de políticas de qualificação da APS, foi com o surgimento dos programas de provimento de âmbito nacional que o capital simbólico da representatividade da APS e do Médico de Família e Comunidade (MFC) ganharam força no debate da categoria médica. Inicialmente com o PROVAB, as entidades médicas - principalmente a Associação Médica Brasileira (AMB) - se contrapuseram à mínima tentativa de regulação estatal no processo de ingresso nas residências médicas, ${ }^{22}$ mas logo foram vencidas pela grande atração do programa nos recém-formados atraídos por este bônus 
para concorrer a vagas de residência e pela possibilidade de um trabalho com boa remuneração e suporte das universidades. ${ }^{23,24}$ Com isso, a APS tornou-se um cenário de trabalho mais atrativo aos médicos recémformados, mesmo que de início apenas de forma transitória.

Na sequência, com o PMM intensificou-se o debate sobre a falta de médicos principalmente na APS e da desassistência principalmente para as populações mais vulneráveis, com embate direto entre a posição do Ministério da Saúde e das entidades médicas. ${ }^{22,25}$ Este embate reverberou para além das entidades, alcançando parte importante da categoria médica e dos profissionais de saúde, bem como para as universidades, gestores, mídia e a população em geral.

A magnitude do programa em seus vários eixos fez com que o debate fosse além do provimento e passasse a discutir questões estruturais, de processo de trabalho e também sobre o perfil do profissional para atuar na APS, entre tantas outras questões debatidas pela sociedade, nas mídias sociais e na mídia tradicional, além da relevância para o meio científico traduzida por dissertações de mestrado, teses de doutorado e artigos nacionais e internacionais.

Portanto, é este cenário fértil de bons resultados do PMM no provimento, ${ }^{26,27}$ da aprovação do programa entre a população, gestores e médicos ${ }^{28-31}$ e o crescente interesse pela residência de MFC $^{32,33}$ e do cenário da APS como campo de trabalho que dá força para legitimar o novo programa no contexto de disputas do novo governo. E, em um cenário de retração de tantas políticas públicas para os mais pobres, ${ }^{9}$ ver surgir um programa que se propõe a fortalecer a APS e a combater iniquidades no provimento de médicos evidencia os acertos do programa anterior e a tentativa de superá-lo.

\section{O Programa Médicos pelo Brasil}

\section{Continuidade do eixo de provimento do PMM}

Assim como o PMM se ancorou na estrutura do PROVAB tentando superar as suas limitações, vemos agora que o PMB se estrutura em alguns acertos do PMM para montar o seu arcabouço de funcionamento e tenta superar alguns dos problemas já evidenciados.

Inicialmente, no novo programa mantém-se o entendimento de que os lugares mais vulneráveis necessitam de suporte do governo federal para o provimento de médicos para o trabalho em seu território e, com isso, mantém-se um número de vagas equivalente ao que existia no PMM. Ressalta-se, entretanto, que as vagas do novo programa não serão novas, mas irão substituir gradativamente as vagas do PMM na medida em que for acabando o contrato em vigência dos médicos em atividade.

\section{Possíveis avanços do PMB}

Uma primeira novidade é a aposta na qualificação dos critérios de vulnerabilidade dos municípios utilizando uma metodologia de classificação proposta pela OCDE na tentativa de atingir municípios prioritários não alcançados pelo PMM e se blindar dos possíveis enfrentamentos com gestores de municípios com maior força política e que poderiam ter se utilizado disso no PMM para negociar um maior número de vagas nas capitais e em locais não prioritários. ${ }^{34} \mathrm{~A}$ aposta parece acertada a priori, mas para ser efetivada precisa resistir às disputas que ainda acontecerão em torno do projeto. 
Uma outra novidade é o modelo de contratação dos médicos. Apesar de se utilizar do artifício criado no PMM da bolsa formação para qualificar os profissionais sem formação direcionada para a APS, verificase que no PMB este processo é temporário e se articula com uma prova de título ao final de 2 anos que validaria ou não a contratação posterior via Consolidação das Leis do Trabalho (CLT) e com um plano de carreira com 4 níveis e progressões a cada 3 anos. No caso de médicos com formação em MFC, o ingresso na carreira seria direto, tendo o mesmo uma atuação de tutor dos médicos em formação. Além disso, o valor da remuneração superior ao PMM e a diversificação desta em componentes fixos e outros variáveis (desempenho, tempo de permanência, distância de grandes centros, etc.) estão entre os elementos propostos pela Organização Mundial da Saúde (OMS) para atração de profissionais. ${ }^{35,36}$

Esta parece ser a grande aposta de atração de médicos do novo programa e se propõe a superar a precarização das relações trabalhistas tão criticada no PMM e na APS brasileira como um todo. ${ }^{37,38}$ Além disso, a medida aponta para um cenário no qual a titulação como MFC para atuar no programa torna-se obrigatória em médio prazo, através da residência médica ou da prova de titulação proposta ao final do período de formação dentro do programa.

Esta obrigatoriedade do título de MFC para efetivar a contratação via CLT pode ser utilizada pelos programas de residência em MFC para atrair candidatos que desejem uma formação mais sólida antes de ingressar no PMB, mas para isso seria interessante que a titulação via residência fosse prioritária em relação à titulação via prova de especialidade para não minar esta potencialidade do programa.

\section{Riscos para o recrutamento e fixação de profissionais}

Entretanto, vale ressaltar que o plano de carreira e o vínculo trabalhista dialoga principalmente com a cobrança das entidades médicas, mas nos estudos de provimento nacionais e internacionais não aparece entre os principais elementos para garantir fixação, já que aumentos na renda não compensariam outras fontes de insatisfação com o trabalho. ${ }^{39,40}$ A flexibilidade do contrato de trabalho tem muito mais importância neste sentido ${ }^{40}$ e não é um dos elementos presentes no PMB, que no início começa com uma carga de 60 horas semanais e depois segue com uma carga horária de 40 horas. Ainda sobre carga horária, o programa atual critica as $32 \mathrm{~h}$ de atendimento no programa anterior, mas nos primeiros 2 anos do novo programa a cada 2 meses o médico fica 1 semana acompanhando o tutor, não havendo tanta diferença na carga horária total, mas com descontinuação do atendimento por 1 semana inteira.

Além disso, um outro elemento destacado na literatura como indutor de fixação de médicos é o suporte educacional e contato com centros formadores ao diminuir a sensação de isolamento. ${ }^{35,36,40-43}$ No PMM este elemento aparecia através da supervisão e dos encontros locorregionais e, mesmo que acontecesse apenas 1 vez ao mês, o seu caráter longitudinal se mostrou associado à intenção de permanência na APS quando havia um bom supervisor. ${ }^{31}$ No novo programa este suporte educacional perdura apenas nos 2 primeiros anos de bolsa formação, podendo após este período levar à sensação de isolamento nos locais mais distantes e, com isso, levar ao abandono do programa. 
Pensando mais especificamente sobre o provimento, um elemento muito discutido na literatura é a atração de médicos de outros paíse ${ }^{35,44}$ e, neste sentido, o PMB aposta na suficiência de médicos brasileiros para preencher suas vagas. Faz isso em uma tentativa simbólica de ruptura total com o PMM ao questionar o modelo de contratação de médicos cubanos via cooperação com a OPAS que pelo PMM já tinha previsão de encerramento em 2019. Entretanto, para que isto fosse possível, o eixo de formação previa abertura de vagas de graduação em medicina e ampliação da residência de MFC ${ }^{45}$ que tiveram seu curso interrompido em 2016, fazendo com que esta aposta seja arriscada e corra-se o risco de manter a desassistência que já existe em muitos municípios desde a saída dos cubanos.

Diante destes elementos, percebe-se que o cenário de atratividade do programa ainda não está dado e precisa se consolidar na prática. Além disso, há risco do programa apenas atrair profissionais que já se encontram trabalhando na APS em contratos precários. Com isso, não haveria acréscimo real de novos médicos para o trabalho na APS e seria importante pensar meios de apoiar medidas regionais de desprecarização das relações de trabalho.

\section{Outros riscos relacionados ao PMB}

O PMM apresentava, além do seu eixo de provimento, dois outros eixos importantes: um de qualificação da estrutura e outro de formação. ${ }^{45} \mathrm{O}$ eixo de qualificação da estrutura previa financiamento para reformas e ampliações das unidades básicas de saúde (UBS) e conseguiu contribuir para qualificação estrutural das UBS que recebiam médicos do PMM. ${ }^{31,46,47}$ Já o eixo formativo previa ações de reorientação da formação médica com ampliação e interiorização das vagas de graduação e residência médica como dispositivo de formação de profissionais de acordo com as necessidades do Sistema Único de Saúde (SUS), tendo conseguido resultados importantes na interiorização dos cursos médicos, reorientação dos currículos de medicina, além da ampliação e ocupação das vagas de residência de MFC. 32,33,48

Já para o PMB não foi apresentado nenhum elemento que desse seguimento ao incentivo e financiamento da qualificação estrutural das UBS. Com relação ao eixo formativo, há apenas a referência a uma especialização nos primeiros 2 anos do programa que levaria à possibilidade de titulação em MFC após aprovação na prova de título. Entretanto, não houve referência a elementos de reorientação da formação médica para o cenário da APS e nem de estímulo a residência de MFC, correndo-se o risco inclusive do PMB vir a competir pelo interesse de egressos dos cursos médicos em fazer a residência de MFC. Seria, portanto, interessante, desde já, pensar formas de valorizar a residência de MFC em comparação ao título para não haver um esvaziamento da procura pela residência de MFC.

Antes de encerrar esta análise, chama atenção ainda o foco da medida provisória na criação da Agência para o Desenvolvimento da Atenção Primária à Saúde (Adaps) como forma de tenta driblar as dificuldades burocráticas existentes no serviço público, mas, assim como no caso da criação da EBSERH, abre-se um espaço potencial para a privatização do SUS. ${ }^{49,50}$ Isto se torna ainda mais preocupante quando observamos que a medida não prevê em seu conselho deliberativo representação dos usuários do SUS ou pelo menos do Conselho Nacional de Saúde, mas há representação efetiva de entidades privadas do setor saúde. ${ }^{2}$ 


\section{Considerações finais}

O PMB, ao tentar superar limitações do PMM, traz como principal medida a proposta de uma carreira médica para atuação na APS, sem, no entanto, apresentar outros elementos importantes para a fixação profissional. Desta forma, apresenta-se inicialmente como uma política mais frágil que seu antecessor, tendo como foco apenas o provimento de médicos, correndo o risco de não atingir os objetivos a que se propôs enquanto política pública e, através da Adaps, abrir margem para a privatização dos serviços de APS e do SUS como um todo.

Destaca-se ainda que o programa não supera um dos principais problemas do PMM de focar apenas na atração e qualificação do médico ao invés de investir na qualificação de toda a equipe de saúde, podendo, inclusive, intensificar disparidades importantes de remuneração e valorização entre os diversos profissionais.

Com isso, fica evidente a importância de debater publicamente as medidas já apresentadas pelo PMB como forma de contribuir para a sua qualificação durante a tramitação da MP no Congresso Nacional.

\section{Contribuição dos autores}

Concepção e/ou delineamento do estudo: AJMN, DSB; Redação preliminar: AJMN, DSB; Revisão crítica da versão preliminar: AJMN, DSB; Aprovação final da versão a ser publicada: AJMN, DSB.

Todos os autores aprovaram a versão final e concordaram com prestar contas sobre todos os aspectos do trabalho.

\section{Conflito de interesses}

Declaram não haver.

\section{Referências}

1. Brasil. Lei $\mathrm{N}^{\circ} 12.871$, de 22 de outubro de 2013. Institui o Programa Mais Médicos, altera as Leis $\mathrm{N}^{\circ} 8.745$, de 9 de dezembro de 1993, e N 6.932, de 7 de julho de 1981, e dá outras providências. [Internet]. Brasília: Diário Oficial da União; 2013. Disponível em: http://www.planalto.gov.br/ccivil_03/_Ato2011-2014/2013/Lei/L12871.htm

2. Brasil. Medida Provisória No 890, de 10 de agosto de 2019. Institui o Programa Médicos pelo Brasil, no âmbito da atenção primária à saúde no Sistema Único de Saúde, e autoriza o Poder Executivo federal a instituir serviço social autônomo denominado Agência para o Desenvolvimento da Atenção Primária à Saúde. [Internet]. Brasília: Diário Oficial da União; 2019. Disponível em: http://www.in.gov.br/en/web/dou/-/medida-provisoria-n-890-de-1-de-agosto-de-2019-208356620

3. Jesus RA, Medina MG, Prado NMBL. Programa Mais Médicos: análise documental dos eventos críticos e posicionamento dos atores sociais. Interface (Botucatu). 2017;21(Suppl 1):1241-56. https://doi.org/10.1590/1807-57622016.0555

4. Oliveira FP, Costa AM, Cardoso AJC, Trindade JS, Dias IMAV. Análise das emendas parlamentares ao Programa Mais Médicos: o modelo de formação médica em disputa. Saúde Debate. 2017;41(no.spe3):60-73. https://doi.org/10.1590/0103-11042017s305

5. Brasil. Decreto No 9.795, de 17 de maio de 2019. Aprova a Estrutura Regimental e o Quadro Demonstrativo dos Cargos em Comissão e das Funções de Confiança do Ministério da Saúde, remaneja cargos em comissão e funções de confiança, transforma funções de confiança e substitui cargos em comissão do Grupo-Direção e Assessoramento Superiores - DAS por Funções Comissionadas do Poder Executivo - FCPE. [Internet]. Brasília: Diário Oficial da União;2019. Disponível em: http://www.planalto.gov.br/ccivil_03/_ato20192022/2019/decreto/D9795.htm 
6. Araujo RS, Dilligenti MP. A Retomada da Ortodoxia Neoliberal: O Austericídio dos Direitos Sociais no Brasil. In: Anais do $7^{\circ}$ Encontro Internacional de Política Social, 14 Encontro Nacional de Política Social. Tema: Contrarreformas ou Revolução: respostas ao capitalismo em crise; 2019 Jun 3-6; Vitória ES, Brasil. 13 p.

7. Wanderley MB, Sant'Ana RS, Martinelli ML. Os desafios do atual contexto: um diálogo a partir da seguridade. Serv Soc Soc (São Paulo). 2019;(135):207-12. https://doi.org/10.1590/0101-6628.174

8. Doniec K, Dall'Alba R, King L. Austerity threatens universal health coverage in Brazil. Lancet. 2016;388(10047):867-8. https://doi.org/10.1016/S0140-6736(16)31428-3

9. Santos IS, Vieira FS. Direito à saúde e austeridade fiscal: o caso brasileiro em perspectiva internacional. Ciênc Saúde Coletiva. 2018;23(7):2303-14. https://doi.org/10.1590/1413-81232018237.09192018

10. Brasil. Ministério da Saúde. Secretaria de Atenção à Saúde. Departamento de Atenção Básica. Política Nacional de Atenção Básica. (Pactos pela Saúde). Brasília: Ministério da Saúde; 2006.

11. Batista CB. Movimentos de reorientação da formação em saúde e as iniciativas ministeriais para as universidades. Barbaroi. 2013;38:97125.

12. Brasil. Ministério da Saúde. Departamento de Atenção Básica. Manual de Estrutura Física das Unidades Básicas de Saúde: Saúde da Família. Brasília: Ministério da Saúde; 2006.

13. Neves RG, Duro SMS, Muñiz J, Castro TRP, Facchini LA, Tomasi E. Estrutura das unidades básicas de saúde para atenção às pessoas com diabetes: Ciclos I e II do Programa Nacional de Melhoria do Acesso e da Qualidade. Cad Saúde Pública. 2018;34(4):e00072317. https://doi.org/10.1590/0102-311x00072317

14.Pinto LF, Giovanella L. Do Programa à Estratégia Saúde da Família: expansão do acesso e redução das internações por condições sensíveis à atenção básica (ICSAB). Ciênc Saúde Coletiva. 2018;23(6):1903-14. https://doi.org/10.1590/1413-81232018236.05592018

15. Bastos ML, Menzies D, Hone T, Dehghani K, Trajman A. The impact of the Brazilian family health on selected primary care sensitive conditions: A systematic review. PLoS One. 2017;12(8):e0182336. https://doi.org/10.1371/journal.pone.0182336

16. Maia LG, Silva LAD, Guimarães RA, Pelazza BB, Pereira ACS, Rezende WL, et al. Hospitalizations due to primary care sensitive conditions: an ecological study. Rev Saúde Pública. 2018;53:1-10. https://doi.org/10.11606/s1518-8787.2019053000403

17. Brasil VP, Costa JSD. Hospitalizações por condições sensíveis à atenção primária em Florianópolis, Santa Catarina - estudo ecológico de 2001 a 2011. Epidemiol Serv Saúde. 2016;25(1):75-84. https://doi.org/10.5123/s1679-49742016000100008

18. Aquino R, de Oliveira NF, Barreto ML. Impact of the family health program on infant mortality in Brazilian municipalities. Am J Public Health. 2009;99(1):87-93. https://doi.org/10.2105/AJPH.2007.127480

19. Carvalho SC, Mota E, Dourado I, Aquino R, Teles C, Medina MG. Hospitalizations of children due to primary health care sensitive conditions in Pernambuco State, Northeast Brazil. Cad Saúde Pública. 2015;31(4):744-54. https://doi.org/10.1590/0102-311X00069014

20. Rasella D, Harhay MO, Pamponet ML, Aquino R, Barreto ML. Impact of primary health care on mortality from heart and cerebrovascular diseases in Brazil: a nationwide analysis of longitudinal data. BMJ. 2014;349:g4014. https://doi.org/10.1136/bmj.g4014

21. Afonso MPD, Shimizu HE, Merchan-Hamann E, Ramalho WM, Afonso T. Association between hospitalisation for ambulatory caresensitive conditions and primary health care physician specialisation: a cross-sectional ecological study in Curitiba (Brazil). BMJ Open. 2017;7(12):e015322. https://doi.org/10.1136/bmjopen-2016-015322

22. Gomes LB. A atual configuração política dos médicos brasileiros: uma análise da atuação das entidades médicas nacionais e do movimento médico que operou por fora delas [Tese de doutorado]. Rio de Janeiro: Universidade Federal do Rio de Janeiro, Faculdade de Medicina; 2016.

23. Oliveira CM, Cruz MM, Kanso S, Reis AC, Lima A, Torres RMC, et al. Avaliabilidade do Programa de Valorização do Profissional da Atenção Básica (PROVAB): desafios para gestão do trabalho. Ciênc Saúde Coletiva. 2015;20(10):2999-3010. https://doi.org/10.1590/1413-812320152010.13322014

24. Araújo CA, Michelotti FC, Ramos TKS. Programas governamentais de provisão: perfil e motivações dos médicos que migraram do Programa de Valorização do Profissional da Atenção Básica (Provab) para o Mais Médicos em 2016. Interface (Botucatu). 2017;21(Suppl 1):1217-28. https://doi.org/10.1590/1807-57622016.0607 
25. Gomes LB, Merhy EE. Uma análise da luta das entidades médicas brasileiras diante do Programa Mais Médicos. Interface (Botucatu). 2017;21(Suppl 1):1103-14. https://doi.org/10.1590/1807-57622016.0363

26. Rech MRA, Hauser L, Wollmann L, Roman R, Mengue SS, Kemper ES, et al. Qualidade da atenção primária à saúde no Brasil e associação com o Programa Mais Médicos. Rev Panam Salud Publica. 2018;42:e164. https://doi.org/10.26633/RPSP.2018.164

27. Kemper ES, Tasca R, Harzheim E, Suárez Jiménez JM, Hadad J, Sousa MF. Cobertura universal em saúde e o Programa Mais Médicos no Brasil. Rev Panam Salud Publica. 2018;42:e1. https://doi.org/10.26633/RPSP.2018.1

28. Comes Y, Trindade JS, Shimizu HE, Hamann EM, Bargioni F, Ramirez L, et al. Avaliação da satisfação dos usuários e da responsividade dos serviços em municípios inscritos no Programa Mais Médicos. Ciênc Saúde Coletiva. 2016;21(9):2749-59. https://doi.org/10.1590/141381232015219.16202016

29. Santos MAM, Souza EG, Cardoso JC. Avaliação da Qualidade da Estratégia Saúde da Família e do Programa Mais Médicos na Área Rural de Porto Velho, Rondônia. Rev Eletr Gest Soc. 2016;10(26):1327-46. https://doi.org/10.21171/ges.v10i26.2089

30. Pinto HA, Oliveira FP, Santana JSS, Santos FOS, Araujo SQ, Figueiredo AM, et al. Programa Mais Médicos: avaliando a implantação do Eixo Provimento de 2013 a 2015. Interface (Botucatu). 2017;21(Suppl 1):1087-101. https://doi.org/10.1590/1807-57622016.0520

31. Melo Neto AJ. Fatores Associados à Intenção de Permanecer Trabalhando na Atenção Primária à Saúde Entre os Médicos Brasileiros do Programa Mais Médicos na Paraíba [Dissertação]. João Pessoa: Universidade Federal da Paraíba; 2019.

32. Oliveira FP, Araújo CA, Torres OM, Figueiredo AM, Souza PA, Oliveira FA, et al. The More Doctors Program and the rearrangement of medical residency education focused on Family and Community Medicine. Interface (Botucatu). 2019;23(Suppl 1):e180008. https://doi.org/10.1590/interface.180008

33. Barrêto DS, Melo Neto AJ, Figueiredo AM, Sampaio J, Gomes LB, Soares RS. The More Doctors Program and Family and Community Medicine residencies: articulated strategies of expansion and interiorization of medical education. Interface (Botucatu). 2019;23(Suppl 1):e180032. https://doi.org/10.1590/interface.180032

34. Oliveira JPA, Sanchez MN, Santos LMP. O Programa Mais Médicos: provimento de médicos em municípios brasileiros prioritários entre 2013 e 2014. Ciênc Saúde Coletiva. 2016;21(9):2719-27. https://doi.org/10.1590/1413-81232015219.17702016

35. Organização Mundial da Saúde (OMS). Trabalhando juntos pela saúde: Relatório Mundial de Saúde 2006. [Internet]. Brasília: Ministério da Saúde; 2007 [citado 2019 Abr 1]. Disponível em: https://www.who.int/whr/2006/06_overview_pr.pdf?ua=1

36. World Health Organization (WHO). Increasing access to health workers in remote and rural areas through improved retention: global policy recommendations. Geneva: World Health Organization; 2010. 72 p.

37. Jardim GS, Oliveira DCS, Montandon DS, Santiago LP, Silva RLAM, Silva IOAM, et al. Índice de satisfação no trabalho de profissionais da Estratégia de Saúde da Família. Rev Eletr Ac Saúde. 2018;10(4):2055-64. https://doi.org/10.25248/REAS411_2018

38. Lima L, Pires DEP, Forte ECN, Medeiros F. Satisfação e insatisfação no trabalho de profissionais de saúde da atenção básica. Esc Anna Nery 2014;18(1):17-24.

39. Campos CVA, Malik AM. Satisfação no trabalho e rotatividade dos médicos do Programa de Saúde da Família. Rev Adm Pública. 2008;42(2):347-68. https://doi.org/10.1590/S0034-76122008000200007

40. Marchand C, Peckham S. Addressing the crisis of GP recruitment and retention: a systematic review. Br J Gen Pract. 2017;67(657):e22737. https://doi.org/10.3399/bjgp17X689929

41. Verma P, Ford JA, Stuart A, Howe A, Everington S, Steel N. A systematic review of strategies to recruit and retain primary care doctors. BMC Health Serv Res. 2016;16:126. https://doi.org/10.1186/s12913-016-1370-1

42. Luna WF, Ávila BT, Brazão CFF, Freitas FPP, Cajado LCS, Bastos LOA. Project More Doctors for Brazil in remote areas of the state of Roraima: relationship between doctors and the Special Supervision Group. Interface (Botucatu). 2019;23(Suppl 1):e180029. https://doi.org/10.1590/interface.180029

43. White CD, Willett K, Mitchell C, Constantine S. Making a difference: education and training retains and supports rural and remote doctors in Queensland. Rural Remote Health. 2007;7(2):700. https://doi.org/10.22605/RRH700

44. Oliveira FP, Vanni T, Pinto HA, Santos JTR, Figueiredo AM, Araújo SQ, et al. Mais Médicos: um programa brasileiro em uma perspectiva internacional. Interface (Botucatu). 2015;19(54):623-34. DOI: https://doi.org/10.1590/1807-57622014.1142 
45. Brasil. Ministério da Saúde. Secretaria de Gestão do Trabalho e da Educação na Saúde. Programa Mais Médicos - Dois anos: Mais Saúde para os Brasileiros. Brasília: Ministério da Saúde; 2015.

46. Mota RG, Barros NF. O Programa Mais Médicos no Estado de Mato Grosso, Brasil: uma análise de implementação. Ciênc Saúde Coletiva. 2016;21(9):2879-88. https://doi.org/10.1590/1413-81232015219.14582016

47. Soares Neto JJ, Machado MH, Alves CB. O Programa Mais Médicos, a infraestrutura das Unidades Básicas de Saúde e o Índice de Desenvolvimento Humano Municipal. Ciênc Saúde Coletiva.2016;21(9):2709-18. https://doi.org/10.1590/1413-81232015219.16432016

48. Oliveira FP, Pinto HA, Figueiredo AM, Cyrino EG, Oliveira Neto AV, Rocha VXM. Brazilian More Doctors Program: assessing the implementation of the Education Axis from 2013 to 2015. Interface (Botucatu). 2019;23(Suppl 1):e170949. DOI: https://doi.org/10.1590/ interface.170949

49. Lima JB. A contrarreforma do Sistema Único de Saúde: o caso das Organizações Sociais. Argumentum. 2018;10(1):88-101. https://doi.org/10.18315/argumentum.v10i1.18734

50. Silva MD, Clementino MO, Silva AX. A Política de Saúde no Contexto de Contrarreforma do Estado Brasileiro e os Novos Modelos de Gestão: análise da Empresa Brasileira de Serviços Hospitalares (EBSERH). In: Saúde em Tempos de Retrocessos e Retirada de Direitos. In: Anais do 7o Seminário Frente Nacional Contra a Privatização da Saúde; 2017 Out 27-29; Maceió, AL, Brasil [citado 2019 Ago 13 ]. Disponível em: http://www.seer.ufal.br/index.php/anaisseminariofncps/article/viewFile/3979/2813 